\title{
Correction to: A stepwise strategy to distinguish menstrual blood from peripheral blood by Fisher's discriminant function
}

\author{
Hongxia $\mathrm{He}^{1,2} \cdot$ Anquan $\mathrm{Ji}^{1} \cdot$ Yixia Zhao $^{1} \cdot \mathrm{Na} \mathrm{Han}^{3}$. Sheng $\mathrm{Hu}^{1}$ - Qinglan Kong ${ }^{4} \cdot \mathrm{Li} \mathrm{Jiang}^{1} \cdot$ Jian $\mathrm{Ye}^{1} \cdot \mathrm{Yao} \mathrm{Liu}^{1,2}$. \\ Qifan Sun ${ }^{1}$ (1)
}

Published online: 7 March 2022

○) Springer-Verlag GmbH Germany, part of Springer Nature 2022

\section{Correction to: International Journal of Legal Medicine (2020) 134:845-851 https://doi.org/10.1007/s00414-019-02196-w}

The authors regret the following errors found after publication of the article:

Table 1: In the first column "MiRNA", "has-" must read "hsa-", "has-miR-451" must read "hsa-miR-451a", "hasmiR-203-3p" must read "hsa-miR-203a-3p", and in the third row of the fourth column, "MI0000283" should be "MIMAT0000264", and the annotation " $n$, sample number" at the bottom of the table should be deleted.

The corrected Table 1 is shown here:

Table 2 In the third row of the second column, "Nonblood sample $(n=20)$ " must read "Blood sample $(n=20)$ "

The corrected Table 2 is shown here:

"Materials and methods": In paragraph "qPCR", "QuantStudio ${ }^{\text {TM }}$ Real-Time PCR software v1.3" must read "HID Real-Time PCR Analysis Software v1.3" instead.
The original article can be found online at https://doi.org/10.1007/ s00414-019-02196-w.

Yao Liu

liuyaol123@yahoo.cn

Qifan Sun

sunqifan@cifs.gov.cn

1 MPS's Key Laboratory of Forensic Genetics, National Engineering Laboratory for Crime Scene Evidence Investigation and Examination, Institute of Forensic Science, Ministry of Public Security (MPS), Beijing 100038, China

2 Faculty of Forensic Sciences, Shanxi Medical University, Taiyuan 030001, Shanxi, People's Republic of China

3 Chinese Center For Disease Control And Prevention, State Key Laboratory of Infectious Disease Prevention and Control, Beijing 102206, China

4 Faculty of Mathematics and Statistics, Zaozhuang University, Zaozhuang 277160, Shandong, People's Republic of China
"Results": In paragraph "A discriminant function to distinguish blood samples from non-blood samples" it must read instead:

$$
\mathrm{Z} 1=-1.772 \Delta \mathrm{Ct}(\mathrm{miR}-451)-8.179 \text {. }
$$

Publisher's note Springer Nature remains neutral with regard to jurisdictional claims in published maps and institutional affiliations. 
Table 1 Information for the four miRNA markers and the internal reference gene

\begin{tabular}{llll}
\hline MiRNA & Mature miRNA sequence & Reference & miRbase annotation \\
\hline hsa-miR-451a & AAACCGUUACCAUUACUGAGUU & {$[9,10,12,14,15,19-21]$} & MIMAT0001631 \\
hsa-miR-214-3p & ACAGCAGGCACAGACAGGCAGU & {$[6,11,17]$} & MIMAT0000271 \\
hsa-miR-203a-3p & GUGAAAUGUUUAGGACCACUAG & {$[6]$} & MIMAT0000264 \\
hsa-miR-205-5p & UCCUUCAUUCCACCGGAGUCUG & {$[7]$} & MIMAT0000266 \\
RNU6b & CTGCGCAAGGATGACACGCAA & {$[7,14,15,19]$} & \\
& ATTCGTAAGCGTTCCATATTTTT & & \\
\hline
\end{tabular}

\begin{tabular}{lcll}
\hline Sample set & Sample type & \multicolumn{2}{l}{ Prediction classification } \\
\cline { 3 - 4 } \cline { 3 - 4 } & Blood sample & $\begin{array}{l}\text { Non-blood } \\
\text { sample }\end{array}$ & Accuracy rate \\
\hline Training set $(n=150)$ & Blood sample $(n=60) 60$ & 0 & $100 \%$ \\
Test set $(n=50)$ & Non-blood sample $(n=90) 0$ & 90 & $100 \%$ \\
& Blood sample $(n=20) 20$ & 0 & $100 \%$ \\
& Non-blood sample $(n=30) 0$ & 30 & $100 \%$ \\
\hline
\end{tabular}

$n$ : sample number 